\title{
Developmental Computational Psychiatry
}

Tobias U. Hauser ${ }^{1,2}$, Geert-Jan Will2,3, Magda Dubois ${ }^{2}$ \& Raymond J. Dolan ${ }^{1,2}$

${ }^{1}$ Wellcome Centre for Human Neuroimaging, University College London, London WC1N 3BG, United Kingdom

${ }^{2}$ Max Planck University College London Centre for Computational Psychiatry and Ageing Research, London WC1B 5EH, United Kingdom

${ }^{3}$ Institute of Psychology, Leiden University, Wassenaarseweg 52, 2333 AK Leiden, The Netherlands

\section{Correspondence}

Tobias U. Hauser

Max Planck UCL Centre for Computational Psychiatry and Ageing Research

Wellcome Centre for Human Neuroimaging

University College London

10-12 Russell Square

London WC1B 5EH

United Kingdom

Phone: +44 / 2076795264

Email: t.hauser@ucl.ac.uk 


\begin{abstract}
Most psychiatric disorders emerge during childhood and adolescence. This is also a period when the brain undergoes substantial growth and reorganisation. However, it remains unclear how a heightened vulnerability to psychiatric disorder relates to brain maturation, and what the underlying mechanisms might be. Here, we propose 'developmental computational psychiatry' as a framework for linking brain maturation to cognitive development. We propose that through modelling some of the brain's fundamental cognitive computations and relating them to brain development, we can bridge the gap between brain and cognitive development. This in turn can lead to a richer understanding of the ontogeny of psychiatric disorders. We illustrate this perspective by taking examples from reinforcement learning (RL) and dopamine function, showing how computational modelling deepens an understanding of how cognitive processes, such as reward learning, effort learning, and social evaluation might go awry in psychiatric disorders. Finally, we formulate testable hypotheses and sketch the potential and limitations of developmental computational psychiatry.
\end{abstract}




\section{The importance of development in psychiatry}

Psychiatric disorders are strongly rooted in development, with most mental health problems emerging during childhood and adolescence (Kessler et al., 2005). This holds for classic developmental psychiatric disorders, such as dyslexia or attention-deficit/hyperactivity disorder (ADHD), but also for disorders that are typically associated with adulthood, such as obsessive-compulsive disorder (OCD), depression or personality disorders. While developmental disorders are typically being diagnosed around school entry, OCD rises during early adolescence, and depression and psychosis during late teenage years (Box 1; Kessler et al., 2005). These well-described temporal patterns underpin the notion that development plays a crucial role in the emergence of psychiatric disorders. Ignoring these sensitive developmental periods for the emergence of psychiatric disorders risks a neglect of critical mechanisms that cause psychiatric disorder.

Despite the relevance of development for psychiatry, the bulk of research is focused on adult disorders such that developmental issues are still left in the shadows. Here, we advocate that characterising developmental trajectories of psychiatric disorders is of utmost importance for understanding the core mechanisms that lead to psychiatric disorders. We start by outlining why an understanding of brain computations is likely to be pivotal for understanding typical and atypical brain development. We then highlight the importance of computational modelling for understanding cognitive development and its relation to brain maturation. We then discuss this in the context of reinforcement learning and the developmental trajectory of brain dopamine function. Finally, we consider the promises and challenges of developmental computational psychiatry. 


\section{The importance of computations in developmental psychiatry}

For decades, neuroscientists have investigated the functional anatomy of cognition, i.e. finding out where in the brain a process takes place. This endeavour has been incredibly successful, showing among a range of key findings that faces consistently activate the fusiform face area (Kanwisher, McDermott, \& Chun, 1997), that working memory maintenance involves dorsolateral prefrontal cortex (Curtis \& D'Esposito, 2003), and that parts of the medial prefrontal cortex responds to errors (Iannaccone et al., 2015). This approach has only had modest success when it comes to understanding psychiatric disorders. In our view this might be related to the issue of asking the wrong question. We suggest that rather than asking where something is impaired in the brain we need instead to ask how it goes awry. In other words we need to understand the computations performed by the brain, and how these computations go wrong.

\section{Computations in the brain}

A fundamental question for understanding brain function is what computations are performed in neuronal populations that support a particular cognitive process. Although cognitive constructs, such as learning, cognitive flexibility or decision making, allow us to understand behaviour at a psychological level, it is unlikely that the brain adheres to this notional taxonomy. Equally, it is unlikely that a single brain region is responsible for a construct such as cognitive flexibility. Instead it is likely that most expressions of cognition are the product of multiple distinct processes that necessitate different computations, in turn executed by separate neural populations. A disordered cognitive flexibility can thus arise from multiple distinct impairments, which may share little more than them contributing to what we call cognitive flexibility. We thus need to try to understand how neuronal populations integrate information to compute what we conceptualise as a cognitive process. Only by parcellating the 
computational mechanisms underlying cognition can we truly understand which processes go awry in pathology.

A promising way to bridge the gap between neural activity and cognition builds on the idea that the brain is a sophisticated information-processing machine. How can we understand such a complex device? One approach is to exploit working principles of artificial intelligence and relate these to brain processes. The goal of artificial intelligence is to create machines capable of learning and reasoning with minimal instruction. This has enabled the creation of powerful algorithms that solve complex tasks, such as playing Chess or Go (Silver et al., 2016) without a necessity to provide detailed prior instruction. We as investigators can take a lead from the algorithms used in artificial intelligence and assess how an artificial agent might solve a task faced by human agents. The predictions from such models can then be used to analyse whether cognitive processes approximate similar principles, and if so, which neural systems support these.

\section{Computational modelling from neurons to cognition}

Computational models for understanding the workings of the brain can broadly be divided into two types of models. One class describes what these neural populations are computing (i.e. the algorithm), and the other explain how such an algorithm is implemented at a neural level (Hauser, Fiore, Moutoussis, \& Dolan, 2016). We can label these classes as algorithmic and implementation models as per the nomenclature proposed by Marr (1982) (Fig. 1). 


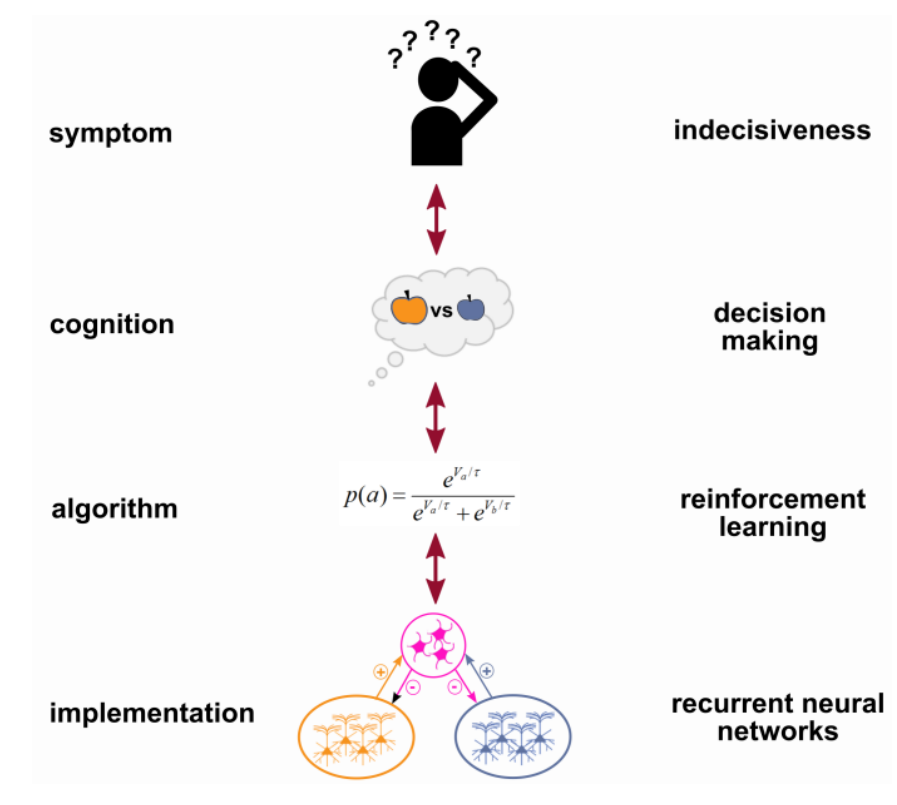

Figure 1. Understanding the neurocomputational mechanisms driving developmental psychiatric symptoms. Computational modelling in developmental psychiatry allows us to understand how the emergence of psychiatric symptoms during development is linked to atypical brain maturation. To bridge the gap between brain (e.g. disrupted dopamine signalling) and symptoms (e.g. indecisiveness or apathy), we can employ computational models. Implementation models simulate neural population activity and make predictions about how aberrant neural function might cause cognitive deficits. Alternatively, we can use algorithmic models that describe the computations underlying cognitive processes. These models allow us to parse a behaviour (e.g. decision making) into its component computations, which allows us to relate them to neural substrates that perform these computations. By connecting brain maturation to the development of specific computational processes, we can understand how aberrant developmental trajectories might lead to psychiatric disorder.

Implementation models describe how (populations of) neurons process information and how they interact in order to solve a task. These models can capture how computations unfold in the brain (e.g., Hunt et al., 2012), but their complexity render it difficult to model more sophisticated aspects of cognition. Although these models have substantial potential for developmental computational psychiatry (e.g., Krystal et al., 2017), in the interest of brevity we focus on algorithmic models alone in the remainder of this article.

Algorithmic models come in various flavours, such as reinforcement learning (Sutton \& Barto, 1998), Bayesian models (Friston et al., 2014), but also deep neural networks (Yamins et al., 2014; Zipser \& Andersen, 1988). An advantage of these models is that they provide a principled understanding of the computational mechanisms that underlie cognitive processes, and thus allow to link cognitive mechanisms to symptoms. One difficulty is that linking these 
computational principles to their neuronal implementation is not always trivial. For illustration, we consider the role of dopamine in learning and why its function is of interest to developmental psychiatry.

\section{Algorithmic models: dopamine in reinforcement learning}

Reinforcement learning (RL) comprises a multitude of models, which adhere to a core principle that agents seek to maximise reward over time (Sutton \& Barto, 1998). RL allows an agent to learn about its environment through a process of trial and error and by so doing the agent can learn to maximise its future reward.

A key aspect of RL (Fig. 2) is the idea that the agent forms predictions about what is going to happen conditional on performing a certain action in a specific environment (state). These predictions are continuously evaluated and refined. To perform this refinement, the agent compares what happened following an action (outcome) with what it had predicted would happen prior to taking the action (expectation). The difference between an experienced outcome and a predicted outcome forms a quantity referred to as a prediction error (PE). The PE indicates whether an outcome is better or worse than expected (positive or negative PE respectively), and how much the expectation deviated from the outcome (magnitude of PE). This PE is used to update future predictions leading to a refinement of the agent's model of the environment. 


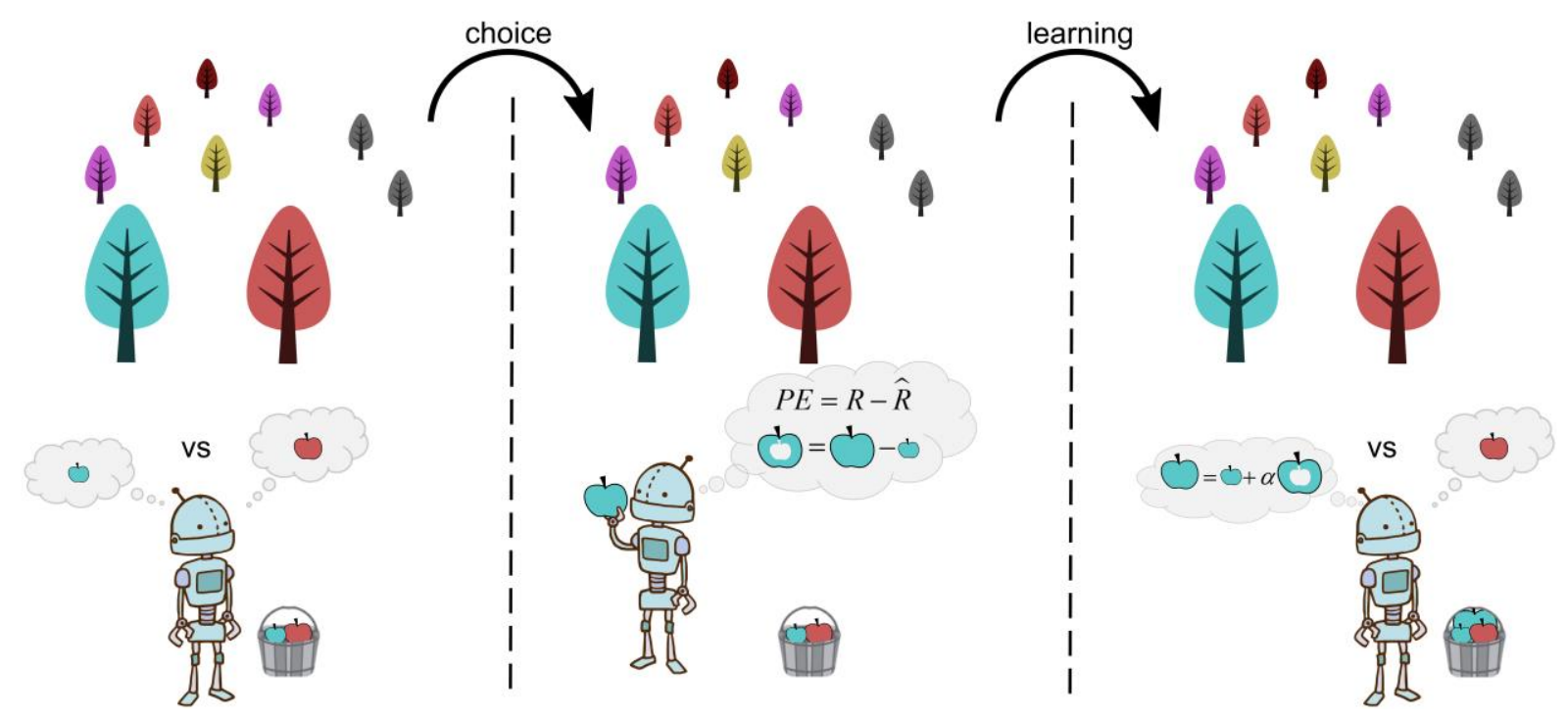

Figure 2. Understanding the neural mechanisms of reinforcement learning. One of the most successfully used algorithms for computational neuroscience is prediction error-based reinforcement learning. To make decisions that lead to a rich harvest, our robot Joko has to try to pick the best (in this case largest) apples. To decide which tree to choose from, he forms an expectation about how good each tree is, based on what he has collected so far (left panel). After he has made a choice (middle), he re-evaluates how good the tree is, based on the size of the currently harvested apple. To do this he computes a prediction error $(P E)$, the difference between what he expected $(R)$ and what he actually got $(R)$. Here, the apple was much larger than expected, which elicited a positive PE. For his subsequent decision (right), he updates his expectation for the blue tree using the PE. In the example, his expectation increased because he experienced a positive PE. The influence of this PE on his expectation is moderated by a learning rate $\alpha$, which determines how much weight to ascribe to current over previous experiences. Through iterative interactions with the environment, PE-based learning allows Joko to converge on the most accurate belief regarding which tree produces the biggest apples. Some of the subfigures are used from freepik.com.

$\mathrm{RL}$ is a method used in artificial intelligence (Sutton \& Barto, 1998), but what does the brain have to do with it? In the mid to late 1990s it was discovered that PE signals had a direct correlate in the brain, where phasic release of dopamine during reward learning accorded with predictions of so-called temporal-difference RL models (Montague, Dayan, \& Sejnowski, 1996; Schultz, Dayan, \& Montague, 1997). Neurons in dopaminergic midbrain (Fig. 3a), located in the substantia nigra and the ventral tegmental area, fired when an outcome was more rewarding than expected (positive PE), but decreased their firing rate when an outcome was worse than expected (negative PE).

Since then, PEs have been found not only in the dopaminergic midbrain, but also in dopamine target regions, such as the ventral striatum (O'Doherty et al., 2004; O'Doherty, 
Dayan, Friston, Critchley, \& Dolan, 2003), which belongs to the mesolimbic dopamine pathway (Fig. 3). Activation in this area increases with a positive reward PE, i.e. if an outcome is more rewarding than anticipated, and decreases when the outcome is less rewarding (i.e. negative PE) (e.g., Rutledge, Dean, Caplin, \& Glimcher, 2010; Hauser, Eldar, \& Dolan, 2017; O'Doherty et al., 2003). Although these human studies use methods (such as functional MRI) which cannot directly measure neurochemicals, such as dopamine, pharmacological manipulations have shown that the PE signal in humans most likely reflects phasic dopamine activity (Pessiglione, Seymour, Flandin, Dolan, \& Frith, 2006), and that dopamine-boosting drugs restore blunted reward PEs seen in older adults (Chowdhury et al., 2013).

\section{Prediction errors beyond reward}

PEs have mainly been investigated in the context of reward learning. More recently, it was discovered that PE-based learning is a more general motif for learning, and is exploited in other domains. Besides reward learning, PE signals are important for learning about pain (Seymour et al., 2004, 2005; Eldar, Hauser, Dayan, \& Dolan, 2016), effort (Hauser, Eldar, et al., 2017), and in social evaluation (Will, Rutledge, Moutoussis, \& Dolan, 2017). Importantly, these PEs appear to be encoded in dopaminergic target regions.

The ventral striatum is a key region for the expression of PEs not only for learning about basic reinforcers such as food rewards, but also about more abstract reinforcers such as social approval (Jones et al., 2011, 2014). Recently, we showed that such striatal PEs not only drive learning about social approval, but also the subjective value people attribute to themselves (i.e., self-esteem) based on learning how much others value them (Fig. 3b; Will et al., 2017). Self-esteem is the subjective valuation of our own worth and is shaped by the appraisals we receive from others (Denissen, Penke, Schmitt, \& van Aken, 2008; Gruenenfelder-Steiger, Harris, \& Fend, 2016; Leary, Tambor, Terdal, \& Downs, 1995). We 
used a social evaluation task wherein subjects received approval and disapproval feedback from strangers (Fig. 3b). We were interested in how social approval PEs affected subjects' selfesteem, by asking them to report their momentary self-esteem. We found that subjects' selfesteem was strongly influenced by social approval PEs, and that updates to a person's selfesteem were encoded in ventromedial prefrontal cortex (vmPFC).

Crucially, in this study people differed in the extent to which social approval PEs determined their self-esteem. People who had greater fluctuations in self-esteem in response to social feedback had enhanced social PE responses in anterior insula and scored higher on a range of psychiatric symptoms. This suggests that a greater sensitivity to social approval PEs represent a neurocomputational marker of psychiatric vulnerability. We discuss the implications of these findings for development and psychiatric disorders in the outlook section.

a

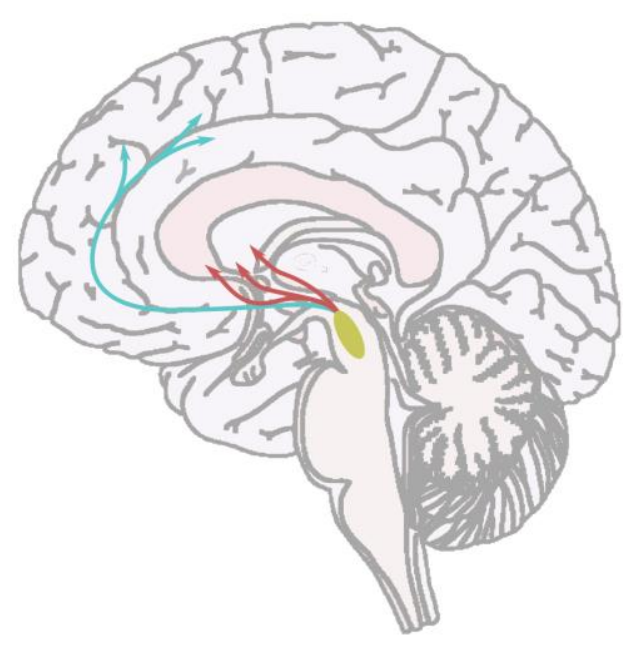

b

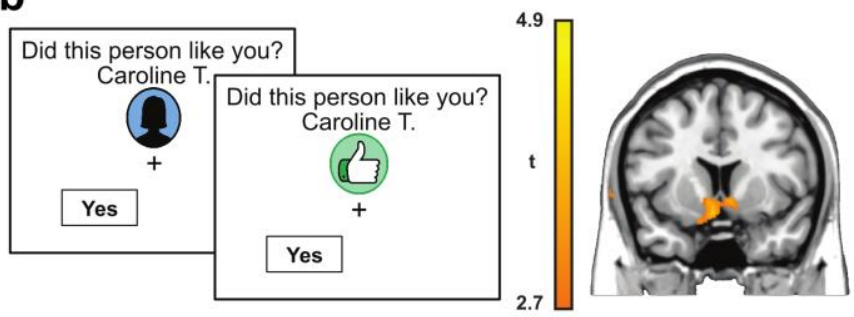

C

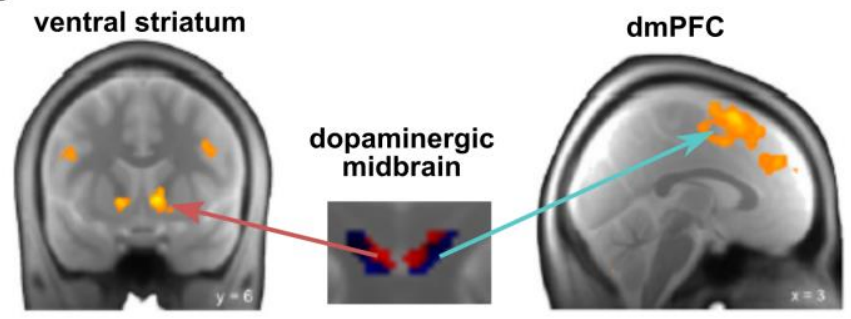

Figure 3. Dopamine pathways in learning. (a) The majority of dopamine neurons are located in dopaminergic midbrain nuclei of ventral tegmental area and the substantia nigra (yellow). Dopamine is then released through several ascending projections. The two main pathways are mesolimbic projections (red) and mesocortical projections (blue). The mesolimbic projections target areas such as the ventral striatum and mature in early adolescence. The mesocortical pathway targets areas of the prefrontal cortex, such as dorsomedial prefrontal cortex (dmPFC), and does not mature until late adolescence. (b) The ventral striatum responds to prediction errors about reward, including social rewards such as approval from another person. (c) Mesolimbic and mesocortical pathways have different functions in learning. While the former is critical for learning about rewards, the latter is implicated in processes such as effort learning. Both learning signals are simultaneously encoded in the dopaminergic midbrain (middle), but show a spatial segregation, where reward PEs are primarily encoded in dorsomedial areas (red) and effort PEs in ventrolateral areas of the midbrain. The distinct developmental trajectories of these pathways (Box 1) pose critical questions about the development of 
the functions they subserve and how they are related to the emergence of psychiatric symptoms, such as apathy. Figures in panel (b) are in part taken from Will et al. (2017) and (c) from Hauser et al. (2017).

The dopamine system not only consists mesolimbic pathways that project to ventral striatum, but also a mesocortical pathway that projects to several prefrontal regions, including dorsomedial prefrontal cortex (dmPFC; Paus, 2001). Given that dmPFC has been found to encode effort associated with a choice option (Croxson, Walton, O'Reilly, Behrens, \& Rushworth, 2009; Kurniawan, Guitart-Masip, Dayan, \& Dolan, 2013; Walton, Bannerman, Alterescu, \& Rushworth, 2003), we speculated that it may also encode an effort PE signal. To formally test this we tasked subjects to learn about the reward and effort associated with a stimulus (Hauser, Eldar, et al., 2017). Subjects had to simultaneously learn both features in order to make good decisions. We found that subjects used PE-like learning mechanisms during reward and effort learning. Using functional MRI, we also found that both types of PEs were encoded in the dopaminergic midbrain (Fig. 3c). However, these PEs seemed to be processed along different pathways, with reward PEs expressed in ventral striatum and effort PEs expressed in dmPFC. We also found that the encoding of these PEs in the latter regions was related to subjects' apathy, a trait characterised by a loss of motivation to exert effort for rewarding outcomes (Marin, 1991).

\section{Computations beyond prediction errors}

Besides simple PE-based learning, other RL-related mechanisms have been discovered in the brain during more complex tasks. For example, when an unobservable model of task structure is guiding behaviour, in alignment with 'model-based' planning (Daw, Gershman, Seymour, Dayan, \& Dolan, 2011; Keramati, Smittenaar, Dolan, \& Dayan, 2016; Wunderlich, Smittenaar, \& Dolan, 2012). Moreover, evidence for forward and backward planning in both spatial and non-spatial tasks suggests that the brain might solve complex, hierarchical planning 
by exploiting core RL principles (Johnson \& Redish, 2007; Kurth-Nelson, Economides, Dolan, \& Dayan, 2016; Wunderlich, Dayan, \& Dolan, 2012).

However, if we want to understand why these computations may change with development, and how this could be relevant for the emergence of psychiatric disorder, we need to understand how neural systems that support these computations develop and mature.

\section{From brain anatomy to cognition: tracing developmental trajectories}

The brain undergoes fundamental structural changes throughout childhood and adolescence, and the developmental trajectories extend into the third and fourth decades of life (Foulkes \& Blakemore, 2018; Giedd et al., 1999, 2015). After an initial increase in gray matter size there is then a relative decrease during adolescence (cf Box 1; Paus, 2010). This decline is thought to be driven by a pruning of cortical connections (Ziegler et al., under review), meaning that neural connections become more selective, possibly supporting a more efficient information-processing capability. In white matter, there is a monotonic increase throughout development, probably driven by axonal myelination (Ziegler et al., under review). Myelination provides insulation for connections between neural populations (Virchow, 1854) and supports fast and reliable information transmission.

Importantly, this structural development is not homogeneous across the entire brain, but rather has specific trajectories in different regions (Gogtay et al., 2004). While primary sensory and motor areas show early maturation, higher cognitive areas of the prefrontal cortex are among the last to fully develop. These distinct developmental trajectories are likely to drive - at least in part - the maturation of distinct computational mechanisms. It is thus of little surprise that motor skills ripen prior to complex reasoning skills. 


\section{Dopamine development}

Although we have a good understanding of how cortical regions mature, we know much less about developmental trajectories of neurotransmitter function, such as dopamine. Given the importance of dopamine in signalling PEs, and its role in reinforcement learning, it is critical to understand its ontogeny. A key challenge in tracing dopamine development is that neural populations that release dopamine - and neurotransmitters in general - are located in small structures in the midbrain. These areas present a challenge using traditional neuroimaging methods (Hämmerer et al., 2018) as they are among the most susceptible to movement and other development-related artefacts (Kasper et al., 2017). A second challenge is that structural integrity of these brainstem nuclei carries little information about the maturity of a system as a whole. The effectiveness of a neurotransmitter system depends on multiple factors, such as the pattern of its branching projections and the configuration of receptors in its target regions.

To obtain a proxy for dopamine development in humans, scientists have turned to its development in rodents. Research here has focused on the development of three aspects of the dopamine system: Development of dopamine neurons, growth of dopamine projections, and density of dopamine receptors (Box 1). Dopamine neurons emerge relatively early in brain development. At 6-8 weeks after conception, the foetal human brain possesses functioning dopamine neurons (Sundström et al., 1993). Thus dopamine neurons are in place at birth, but the system needs at least another two decades to fully mature (Box 1). For dopamine to be effective dopamine-sensitive receptors must be available at target sites. The availability of dopamine receptors, such as D1- and D2-receptors differs substantially between brain regions as a function of maturation stage. In the ventral parts of striatum, dopamine receptor density increases during childhood and peaks around onset of puberty (Caballero, Granberg, \& Tseng, 2016). During adolescence, receptor density in the striatum shrinks again with an overall loss of approximately $25 \%$ of its receptor population (Caballero et al., 2016). 
Dopamine receptors in prefrontal cortex show a later maturation than the striatum. Prefrontal receptor expression does not peak until late adolescence, and then continues to prune well into adulthood (Naneix, Marchand, Di Scala, Pape, \& Coutureau, 2012). These findings highlight that dopamine in limbic areas including ventral striatum have a different developmental trajectory compared to prefrontal cortex. The distinction between striatal and prefrontal dopamine development is particularly pronounced when investigating the maturation of dopamine projections. Dopamine projections to the striatum develop during childhood, suggesting that most of the mesolimbic pathway is in place in early adolescence (Coyle \& Campochiaro, 1976). The mesocortical pathway, however, shows protracted development. Most dopamine projections only reach prefrontal cortex during late adolescence, a process that continues into adulthood (Caballero et al., 2016; Naneix et al., 2012). Some of the projections are connections that originally terminated in the striatum and that during adolescence expand into prefrontal areas, leading to a relative decrease of mesolimbic connections (Reynolds et al., 2018). This makes mesocortical projections the only known longrange connections in the brain that continue their growth throughout adolescence (Hoops \& Flores, 2017). Interestingly, dopamine maturation is also mirrored in structural maturation of these brain regions, where the striatum appears to mature before prefrontal cortex (Giedd et al., 2015).

A caveat to the above concerns extrapolating these dopamine findings to humans. Development is much faster in rodents than in humans (weeks vs. decades), while the mature rodent brain is less complex than the human brain, especially in prefrontal cortex. It thus remains challenging to fully understand dopamine development in humans (cf. Box 2). 


\section{Computational mechanisms in development and psychiatry}

So far, we have discussed why computational modelling is important for understanding how the brain processes information. Moreover, we have seen that the anatomical basis on which these computations build upon changes fundamentally, often continuing into adulthood. In what follows, we will highlight why it is critical to bring these two perspectives together to understand how psychiatric disorders might emerge from atypical brain development.

\section{Development of computational abilities}

Despite the importance of computational mechanisms in development, there is surprisingly little research on how these mechanisms change with age. Decision making and learning are relatively complex cognitive processes and it is reasonable to assume these processes have protracted developmental trajectories (Palminteri, Kilford, Coricelli, \& Blakemore, 2016).

A small number of studies have investigated the development of PE-based learning in the context of reward processing. Using a reward learning task in children, adolescents and adults, Cohen et al. (2010) investigated how reward PEs differ in various phases of development. Interestingly, the authors found a quadratic relationship between age and PE encoding activity in the striatum. This means that adolescents showed the strongest responses for positive reward PEs, stronger than both children and adults (Cohen et al., 2010). This striatal hyper-responsiveness could be modulated by activity in the network wherein it is embedded, such as the connectivity between striatum and vmPFC which appears to strengthen with age (van den Bos, Cohen, Kahnt, \& Crone, 2012). This suggests that striatal hyper-responsivity is downregulated in adulthood via prefrontal control, in line with the general notion that prefrontal cortex acts to balance a mesolimbic dopamine system (Galvan, 2010). Adolescent hypersensitivity not only relates to rewarding stimuli, but also to losses, where enhanced PEs 
in the insula are associated with increased learning for these stimuli (Hauser, Iannaccone, Walitza, Brandeis, \& Brem, 2015; also cf. Davidow, Foerde, Galván, \& Shohamy, 2016). Next to nothing is known about the development of other forms of PE learning, such as social evaluation or effort learning. We highlight below why it is essential to characterise the development of these PEs to understand how psychiatric symptoms emerge.

For more complex aspects of decision making, such as model-based reasoning, some first developmental studies have started to surface (Hartley \& Somerville, 2015; van den Bos, Bruckner, Nassar, Mata, \& Eppinger, 2017). An important aspect of reinforcement learning algorithms is knowing that one's environment has hidden, unobservable connections and inferring these is at the core of 'model-based' reasoning. Studies of model-based decision making indicate this form of reasoning only comes online during adolescence, and does not reach full maturity until early adulthood (Decker, Otto, Daw, \& Hartley, 2016; Potter, Bryce, \& Hartley, 2017).

Another challenging decision making process is to arbitrate between choice options, especially if one has to arbitrate between exploring a novel option and exploiting a well-known option. Humans solve this exploration-exploitation trade-off using different strategies, such information-directed or random exploration (Kidd \& Hayden, 2015; Wilson, Geana, White, Ludvig, \& Cohen, 2014). These exploration strategies show distinct developmental patterns, with younger children using simpler, random exploration while adolescents start using complex information-directed exploration (Somerville et al., 2017).

These findings underline that the development of neuro-computational mechanisms is not a unitary process, but rather the result of multiple developmental trajectories that are at work (cf. Box 1). These trajectories are hypothesised to determine how decisions are made at each specific developmental stage. In order to fully understand how these different 
computational strategies emerge, we need more sophisticated longitudinal investigations of decision making and relate these to structural and functional brain development.

\section{Computational deficits in psychiatric disorders}

Decision making and learning deficits are common across psychiatric disorders (Montague, Dolan, Friston, \& Dayan, 2012). A putative dopamine deficit in several disorders has led to a growing literature on aberrant computations in psychiatry. However, almost no studies have investigated how psychiatric disorders are related to developmental aspects of these deficits. Here, we briefly summarise some key results that are related to PE processing and reinforcement learning.

Impaired reward PE learning has been observed in a number of psychiatric disorders. These include psychosis (Gradin et al., 2011; Murray et al., 2007), alcohol abuse (Reiter et al., 2016), OCD (Hauser, Iannaccone, et al., 2017; Hauser, Eldar, \& Dolan, 2016), ADHD (Hauser et al., 2014), and depression (Gradin et al., 2011; Kumar et al., 2008; though see Rutledge et al., 2017 for contrary findings). However, a relative heterogeneity of where in the brain these impairments were found renders it difficult to understand whether impaired PE-learning forms a general feature of psychopathology, or whether there are distinct impairments in specific disorders. Unified approaches and comparable settings across disorders would therefore be desirable.

The development of such deficits has hardly been studied. In a study of ADHD patients, PE impairments seem to be present already during adolescence, and these deficient learning signals render patients to manifest more exploratory decision making using random exploration (Hauser et al., 2014). Based on knowledge that different forms of exploration have distinct developmental trajectories (Somerville et al., 2017) a critical question arises as to when during development these impairments emerge, and whether ADHD patients show distinct 
developmental trajectories in their exploration strategies compared to typically developing children. This would allow to trace when the developmental trajectories of exploration and PE learning deviate from normal development and how such impaired maturation drives ADHD symptoms.

While PEs involved in reward learning exemplify a relatively basic computation with an early development, it is likely that other computational aspects of decision making lead to deficits only at a later stage. For example, OCD has been associated with deficits in modelbased reasoning (Gillan et al., 2011, 2015; Gillan, Kosinski, Whelan, Phelps, \& Daw, 2016). Knowing that model-based reasoning does not fully develop until adolescence (Decker et al., 2016) renders critical the question as to when this deficit emerges in OCD patients.

\section{The importance of a developmental computational psychiatry}

So far we have highlighted several contexts where mechanistic understanding of aberrant developmental trajectories could provide insight into psychiatric disorders. However, we also believe that developmental computational psychiatry is likely to be even more fundamental for some psychiatric deficits, where aberrant developmental trajectories are likely to be the driving causative factor. We highlight this in two examples, apathy and self-esteem.

\section{Apathy as aberrant maturational separation of dopamine pathways?}

Apathy is characterised by a lack of motivation and an inability to expend effort to realise goal-directed actions (Chong, 2018; Marin, 1991). Apathy is present in several neurological and psychiatric disorders (Pessiglione, Vinckier, Bouret, Daunizeau, \& Le Bouc, 2017), and as a fundamental component of negative symptoms it is a key determinant of longterm disability in schizophrenia (Green, Horan, Barch, \& Gold, 2015). We also know that many 
apathy-related disorders, such as depression or schizophrenia, emerge during adolescence (Kessler et al., 2005). Recent computational theories have characterised apathy as an imbalanced trade-off between reward and effort (Green et al., 2015; Pessiglione et al., 2017). An inflated representation of effort or a diminished expectation of a reward can lead to a belief that an action is not worth carrying out, which in turn leads to apathetic behaviour. We recently showed that apathy is related to how subjects learn about the effort and reward associated with a stimulus. More specifically, we showed that effort and reward PEs overlap in dmPFC to a greater degree in subjects with increased apathy (Hauser, Eldar, et al., 2017). This suggests that the mesolimbic and the mesocortical pathways might be less well differentiated in such subjects and this overlap might bias the representation of expectations for reward and effort.

As seen above, mesocortical projections are among the last brain pathways to mature (Hoops \& Flores, 2017). Only during adolescence do these projections extend from ventral striatum to target prefrontal target areas. This suggests that a spatial separation of effort and reward PEs as seen in adults might not be present before the mesocortical pathway is fully matured. This also hints that apathy might arise if mesocortical growth and signalling goes awry during adolescence, where for example projections meant to target striatum extend into prefrontal areas. Such aberrant growth during adolescence might lead to a failure of segregation of learning signals and lead to an imbalance between striatal and prefrontal dopamine (Elert, 2014).

To test this hypothesis, we need to employ a fully developmental computational psychiatric approach, where we trace learning of reward and effort in a longitudinal fashion using computational neuroimaging. Having characterised a canonical developmental trajectory, we should then assess development in individuals at risk for apathy and ask whether a misguided unfolding in dopaminergic pathways leads to symptoms such as apathy. Although 
such endeavours are complex and resource-intense, without a developmental perspective such putative maladaptive developmental patterns will remain undetected.

\section{Self-esteem instability as vulnerability for adolescent-onset psychiatric disorders}

Low or fragile self-esteem is present in almost every psychiatric disorder. It is a core characteristic of depression (Orth \& Robins, 2013), anxiety (Sowislo \& Orth, 2013), and eating disorders (O'Dea \& Abraham, 2000), all conditions that commonly have their onset in adolescence (Kessler et al., 2005). Adolescence is also the period where we are particularly sensitive to what peers think of us and it is a period when we are more likely to internalize social rejection in our self-view (Rodman, Powers, \& Somerville, 2017). This greater propensity to internalize rejection may be a key driver for the elevated risk of developing mood or anxiety disorders during adolescence (Davey, Yücel, \& Allen, 2008; Sowislo \& Orth, 2013). However, the precise mechanisms that underlie this vulnerability remain to be investigated.

Our study on the influence of social PEs on self-esteem is a potential starting point for exploring such mechanisms (Will et al., 2017). Given that PEs during non-social learning seem overexpressed during adolescence (Cohen et al., 2010; Hauser et al., 2015), a similar overexpression in social PEs might also drive adolescents' greater propensity to internalize rejection. A heightened sensitivity to social PEs might leave adolescents more vulnerable to developing depression when faced with repeated social rejection.

Such insights not only inform our understanding of how psychiatric disorders arise, but can also inform treatment. Depressed adults with unstable self-esteem are more responsive to certain treatments than those with a stable low self-esteem (Roberts, Shapiro, \& Gamble, 1999). Computational modeling could help identify subgroups of depressed adolescents with unstable self-esteem who might benefit more from therapies targeting self-esteem reactivity than other depressed adolescents. Similarly, multiple disorders that first emerge in adolescence (e.g. 
depression, anxiety disorders, eating disorders) share similar vulnerability, including declines in self-esteem preceded by adversity in the interpersonal domain (e.g. peer rejection, romantic breakup) (Allen \& Badcock, 2003; Davey, Yücel, \& Allen, 2008; Sowislo \& Orth, 2013). Consequently, self-esteem instability in the face of rejection might form a vulnerability factor to multiple adolescent-onset disorders.

A developmental perspective is indispensable for a transdiagnostic and neurobiologically-grounded approach to self-esteem. Both negative beliefs about the self and sensitivity to rejection are often a consequence of chronic exposure to early social adversity (Downey, Lebolt, Rincón, \& Freitas, 1998; Ladd \& Troop-Gordon, 2003). The effects of childhood social adversity, such as maltreatment (van Harmelen et al., 2014) or peer rejection (Will, van Lier, Crone, \& Güroğlu, 2016) are thought to leave a lasting imprint on neural responses to rejection. We suggest developmental computational psychiatry offers a unique set of tools to unravel the mechanisms that underlie interactions between these complex features of a person's biography, environment and brain development.

\section{Conclusions}

In this article, we advocate a consilience between the fields of computational psychiatry and developmental neuroscience to establish a field of developmental computational psychiatry. We highlight why such a computationally inspired framework is likely to be critical for understanding how the brain processes information, and how this might go awry during sensitive periods in development. We illustrate how computational mechanisms are likely to follow specific developmental pathways, because the anatomical substrates upon which they are grounded continue to develop throughout the second and early third decade of life. We highlight the potential insights that a developmental perspective on computational psychiatry can provide and how key questions related to psychopathology are only addressable by 
employing a developmental perspective. We believe that developmental computational psychiatry can allow us to begin to unravel why so many psychiatric disorders emerge during this period of development.

\section{Box 1: Trajectories in cognition, brain and psychiatry}

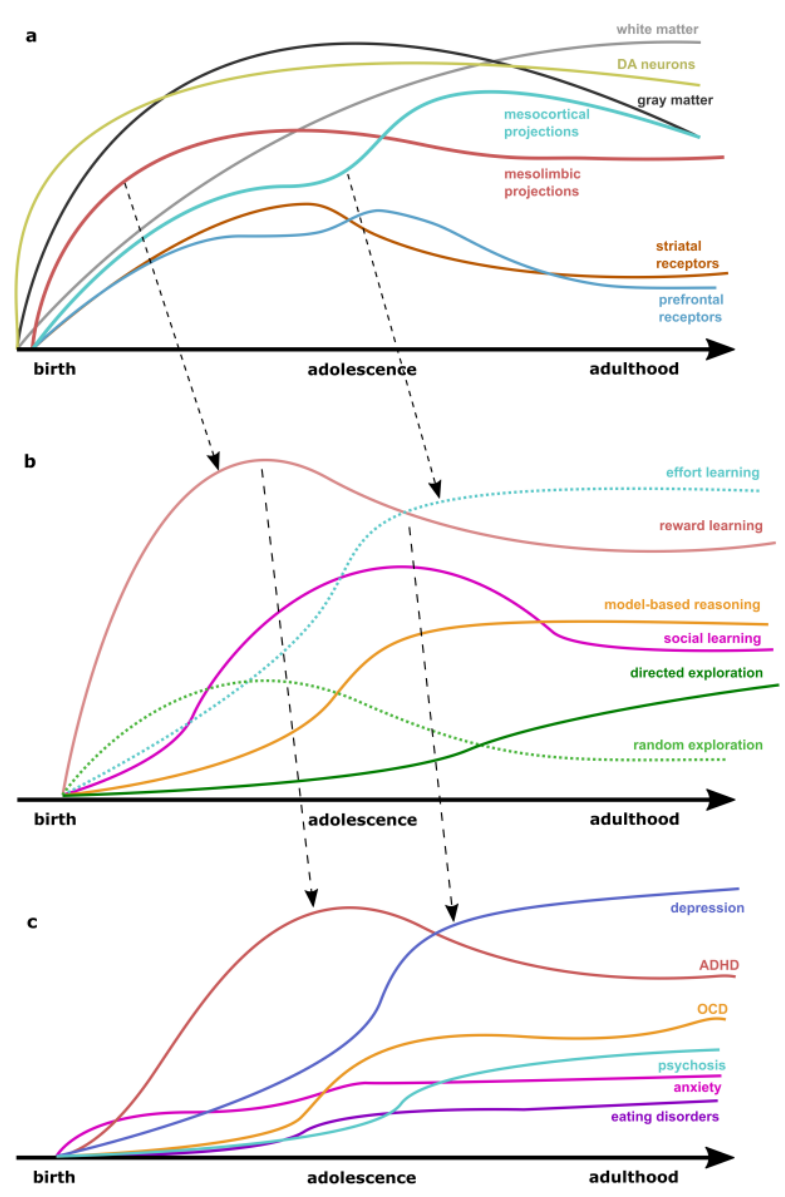

A key challenge in developmental computational psychiatry is to determine how developmental trajectories across multiple systems interact. We need to understand how the development of a neurotransmitter system influences the emergence of a specific cognitive skill, and how an impaired unfolding of this trajectory might promote the emergence of a psychiatric disorder (even decades later in life). A striking feature of brain development (a) is a heterogeneity in developmental trajectories. Although many prefrontal brain areas follow a general pattern of gray and white matter maturation, there is considerable variability both between areas and between individuals. Even within a single neuromodulatory system, such as the dopaminergic system, there are likely to be strikingly different trajectories. For example, dopamine receptors in ventral striatum increase in number mainly during childhood, while mesocortical receptors show a much later maturation. (b) Cognitive functions also show a relative heterogeneity in terms of development. Interestingly, these cognitive trajectories parallel the developmental trajectories of the brain systems with which they are associated (e.g. mesolimbic connections 
and reward learning). (c) To establish a link between brain, computation and the emergence of psychiatric disorders we need to establish normative developmental trajectories and assess when and how they deviate in psychiatric patients. All trajectories are speculative illustrations with dashed lines indicating trajectories that are based little or no empirical data.

\section{Box 2: Challenges for Developmental Computational Psychiatry}

Developmental computational psychiatry faces several challenges, some of which fundamentally limit our abilities to generate novel insight. Here, we provide an incomplete list of what we consider key challenges:

- Understanding the development of neurotransmitters (e.g. dopamine). Understanding this in humans is inherently limited because imaging techniques, such as positron-emissiontomography (PET) are not practical for developmental neuroimaging.

- Sensitive tasks for development. A key to understanding the development of computational mechanisms is to use tasks that measure computations reliably and objectively, and at the same time are viable for research with younger children. To date, most computational tasks have not been systematically evaluated in terms of reliability and other key psychometric criteria, as well as in terms of their sensitivity to computational modelling.

- Longitudinal developmental studies of computational tasks are sparse. We need to rigorously examine how computational mechanisms develop, and overcome limitations in tasks, such as accounting for practice effects, the limitations to use deception in social tasks, or the low reliability of some computational tasks.

- For understanding a cognitive process, we need to have adequate computational models that describe the computations that are carried out by the brain. To overcome these challenges, the field needs a close collaboration between experts in computational 
modelling, computational neuroscience, developmental scientists and experts in psychiatry.

\section{Acknowledgments}

The Wellcome Trust's Cambridge-UCL Mental Health and Neurosciences Network grant (095844/Z/11/Z) supported RJD and TUH. TUH is supported by the Jacobs Foundation. RJD holds a Wellcome Trust Senior Investigator Award (098362/Z/12/Z). GJW is supported by funding from the European Union's Horizon 2020 research and innovation programme under the Marie Skłodowska-Curie grant agreement (No 707404). The Max Planck UCL Centre is a joint initiative supported by UCL and the Max Planck Society. The Wellcome Centre for Human Neuroimaging is supported by core funding from the Wellcome Trust (091593/Z/10/Z). 


\section{References}

Caballero, A., Granberg, R., \& Tseng, K. Y. (2016). Mechanisms contributing to prefrontal cortex maturation during adolescence. Neuroscience and Biobehavioral Reviews, 70, 4-12. https://doi.org/10.1016/j.neubiorev.2016.05.013

Chong, T. T.-J. (2018). Updating the role of dopamine in human motivation and apathy. Current Opinion in Behavioral Sciences, 22, 35-41. https://doi.org/10.1016/j.cobeha.2017.12.010

Chowdhury, R., Guitart-Masip, M., Lambert, C., Dayan, P., Huys, Q., Düzel, E., \& Dolan, R. J. (2013). Dopamine restores reward prediction errors in old age. Nature Neuroscience, 16(5), 648-653. https://doi.org/10.1038/nn.3364

Cohen, J. R., Asarnow, R. F., Sabb, F. W., Bilder, R. M., Bookheimer, S. Y., Knowlton, B. J., \& Poldrack, R. A. (2010). A unique adolescent response to reward prediction errors. Nature Neuroscience, 13(6), 669-671. https://doi.org/10.1038/nn.2558

Coyle, J. T., \& Campochiaro, P. (1976). Ontogenesis of Dopaminergic-Cholinergic Interactions in the Rat Striatum: A Neurochemical Study. Journal of Neurochemistry, 27(3), 673-678. https://doi.org/10.1111/j.1471-4159.1976.tb10393.x

Croxson, P. L., Walton, M. E., O’Reilly, J. X., Behrens, T. E. J., \& Rushworth, M. F. S. (2009). Effort-based cost-benefit valuation and the human brain. The Journal of Neuroscience: The Official Journal of the Society for Neuroscience, 29(14), 4531-4541. https://doi.org/10.1523/JNEUROSCI.4515-08.2009

Curtis, C. E., \& D’Esposito, M. (2003). Persistent activity in the prefrontal cortex during working memory. Trends in Cognitive Sciences, 7(9), 415-423.

Davey, C. G., Yücel, M., \& Allen, N. B. (2008). The emergence of depression in adolescence: development of the prefrontal cortex and the representation of reward. Neuroscience and Biobehavioral Reviews, $\quad 32(1), \quad 19$. https://doi.org/10.1016/j.neubiorev.2007.04.016 
Davidow, J. Y., Foerde, K., Galván, A., \& Shohamy, D. (2016). An Upside to Reward Sensitivity: The Hippocampus Supports Enhanced Reinforcement Learning in Adolescence. Neuron, 92(1), 93-99. https://doi.org/10.1016/j.neuron.2016.08.031

Daw, N. D., Gershman, S. J., Seymour, B., Dayan, P., \& Dolan, R. J. (2011). Model-based influences on humans' choices and striatal prediction errors. Neuron, 69(6), 12041215. https://doi.org/10.1016/j.neuron.2011.02.027

Decker, J. H., Otto, A. R., Daw, N. D., \& Hartley, C. A. (2016). From Creatures of Habit to Goal-Directed Learners: Tracking the Developmental Emergence of Model-Based Reinforcement Learning. Psychological Science, 27(6), 848-858. https://doi.org/10.1177/0956797616639301

Denissen, J. J. A., Penke, L., Schmitt, D. P., \& van Aken, M. A. G. (2008). Self-esteem reactions to social interactions: evidence for sociometer mechanisms across days, people, and nations. Journal of Personality and Social Psychology, 95(1), 181-196. https://doi.org/10.1037/0022-3514.95.1.181

Downey, G., Lebolt, A., Rincón, C., \& Freitas, A. L. (1998). Rejection sensitivity and children's interpersonal difficulties. Child Development, 69(4), 1074-1091.

Eldar, E., Hauser, T. U., Dayan, P., \& Dolan, R. J. (2016). Striatal structure and function predict individual biases in learning to avoid pain. Proceedings of the National Academy of Sciences of the United States of America, 113(17), 4812-4817. https://doi.org/10.1073/pnas.1519829113

Elert, E. (2014). Aetiology: Searching for schizophrenia's roots. Nature, 508(7494), S2-3. https://doi.org/10.1038/508S2a

Foulkes, L., \& Blakemore, S.-J. (2018). Studying individual differences in human adolescent brain development. Nature Neuroscience. https://doi.org/10.1038/s41593-018-0078-4 
Friston, K., Schwartenbeck, P., FitzGerald, T., Moutoussis, M., Behrens, T., \& Dolan, R. J. (2014). The anatomy of choice: dopamine and decision-making. Philosophical Transactions of the Royal Society of London. Series B, Biological Sciences, 369(1655). https://doi.org/10.1098/rstb.2013.0481

Galvan, A. (2010). Adolescent development of the reward system. Frontiers in Human Neuroscience, 4, 6. https://doi.org/10.3389/neuro.09.006.2010

Giedd, J. N., Blumenthal, J., Jeffries, N. O., Castellanos, F. X., Liu, H., Zijdenbos, A., ... Rapoport, J. L. (1999). Brain development during childhood and adolescence: a longitudinal MRI study. Nature Neuroscience, 2(10), 861-863. https://doi.org/10.1038/13158

Giedd, J. N., Raznahan, A., Alexander-Bloch, A., Schmitt, E., Gogtay, N., \& Rapoport, J. L. (2015). Child Psychiatry Branch of the National Institute of Mental Health Longitudinal Structural Magnetic Resonance Imaging Study of Human Brain Development. Neuropsychopharmacology, 40(1), 43-49. https://doi.org/10.1038/npp.2014.236

Gillan, C. M., Apergis-Schoute, A. M., Morein-Zamir, S., Urcelay, G. P., Sule, A., Fineberg, N. A., ... Robbins, T. W. (2015). Functional neuroimaging of avoidance habits in obsessive-compulsive disorder. The American Journal of Psychiatry, 172(3), 284-293. https://doi.org/10.1176/appi.ajp.2014.14040525

Gillan, C. M., Kosinski, M., Whelan, R., Phelps, E. A., \& Daw, N. D. (2016). Characterizing a psychiatric symptom dimension related to deficits in goal-directed control. ELife, 5 . https://doi.org/10.7554/eLife.11305

Gillan, C. M., Papmeyer, M., Morein-Zamir, S., Sahakian, B. J., Fineberg, N. A., Robbins, T. W., \& de Wit, S. (2011). Disruption in the balance between goal-directed behavior and habit learning in obsessive-compulsive disorder. The American Journal of Psychiatry, 168(7), 718-726. https://doi.org/10.1176/appi.ajp.2011.10071062 
Gogtay, N., Giedd, J. N., Lusk, L., Hayashi, K. M., Greenstein, D., Vaituzis, A. C., ... Thompson, P. M. (2004). Dynamic mapping of human cortical development during childhood through early adulthood. Proceedings of the National Academy of Sciences of the United States of America, 101(21), 8174-8179. https://doi.org/10.1073/pnas.0402680101

Gradin, V. B., Kumar, P., Waiter, G., Ahearn, T., Stickle, C., Milders, M., ... Steele, J. D. (2011). Expected value and prediction error abnormalities in depression and schizophrenia. Brain: A Journal of Neurology, 134(Pt 6), 1751-1764. https://doi.org/10.1093/brain/awr059

Green, M. F., Horan, W. P., Barch, D. M., \& Gold, J. M. (2015). Effort-Based Decision Making: A Novel Approach for Assessing Motivation in Schizophrenia. Schizophrenia Bulletin, sbv071. https://doi.org/10.1093/schbul/sbv071

Gruenenfelder-Steiger, A. E., Harris, M. A., \& Fend, H. A. (2016). Subjective and objective peer approval evaluations and self-esteem development: A test of reciprocal, prospective, and long-term effects. Developmental Psychology, 52(10), 1563-1577. https://doi.org/10.1037/dev0000147

Hämmerer, D., Callaghan, M. F., Hopkins, A., Kosciessa, J., Betts, M., Cardenas-Blanco, A., ... Düzel, E. (2018). Locus coeruleus integrity in old age is selectively related to memories linked with salient negative events. Proceedings of the National Academy of Sciences of the United States of America. https://doi.org/10.1073/pnas.1712268115

Hartley, C. A., \& Somerville, L. H. (2015). The neuroscience of adolescent decision-making. Current Opinion in Behavioral Sciences, 5, 108-115. https://doi.org/10.1016/j.cobeha.2015.09.004 
Hauser, T. U., Eldar, E., \& Dolan, R. J. (2016). Neural mechanisms of harm-avoidance learning: A model for obsessive-compulsive disorder? JAMA Psychiatry. https://doi.org/10.1001/jamapsychiatry.2016.1938

Hauser, T. U., Eldar, E., \& Dolan, R. J. (2017). Separate mesocortical and mesolimbic pathways encode effort and reward learning signals. Proceedings of the National Academy of Sciences, 201705643. https://doi.org/10.1073/pnas.1705643114

Hauser, T. U., Fiore, V. G., Moutoussis, M., \& Dolan, R. J. (2016). Computational Psychiatry of ADHD: Neural Gain Impairments across Marrian Levels of Analysis. Trends in Neurosciences. https://doi.org/10.1016/j.tins.2015.12.009

Hauser, T. U., Iannaccone, R., Ball, J., Mathys, C., Brandeis, D., Walitza, S., \& Brem, S. (2014). Role of the Medial Prefrontal Cortex in Impaired Decision Making in Juvenile Attention-Deficit/Hyperactivity Disorder. JAMA Psychiatry.

Hauser, T. U., Iannaccone, R., Dolan, R. J., Ball, J., Hättenschwiler, J., Drechsler, R., ... Brem, S. (2017). Increased fronto-striatal reward prediction errors moderate decision making in obsessive-compulsive disorder. Psychological Medicine, 1-13. https://doi.org/10.1017/S0033291716003305

Hauser, T. U., Iannaccone, R., Walitza, S., Brandeis, D., \& Brem, S. (2015). Cognitive flexibility in adolescence: Neural and behavioral mechanisms of reward prediction error processing in adaptive decision making during development. NeuroImage, 104, 347-354. https://doi.org/10.1016/j.neuroimage.2014.09.018

Hoops, D., \& Flores, C. (2017). Making Dopamine Connections in Adolescence. Trends in Neurosciences, 40(12), 709-719. https://doi.org/10.1016/j.tins.2017.09.004

Hunt, L. T., Kolling, N., Soltani, A., Woolrich, M. W., Rushworth, M. F. S., \& Behrens, T. E. J. (2012). Mechanisms underlying cortical activity during value-guided choice. Nature Neuroscience, 15(3), 470-476, S1-3. https://doi.org/10.1038/nn.3017 
Iannaccone, R., Hauser, T. U., Staempfli, P., Walitza, S., Brandeis, D., \& Brem, S. (2015). Conflict monitoring and error processing: New insights from simultaneous EEG-fMRI. NeuroImage. https://doi.org/10.1016/j.neuroimage.2014.10.028

Johnson, A., \& Redish, A. D. (2007). Neural Ensembles in CA3 Transiently Encode Paths Forward of the Animal at a Decision Point. Journal of Neuroscience, 27(45), 1217612189. https://doi.org/10.1523/JNEUROSCI.3761-07.2007

Jones, R. M., Somerville, L. H., Li, J., Ruberry, E. J., Libby, V., Glover, G., .. Casey, B. J. (2011). Behavioral and neural properties of social reinforcement learning. The Journal of Neuroscience: The Official Journal of the Society for Neuroscience, 31(37), 1303913045. https://doi.org/10.1523/JNEUROSCI.2972-11.2011

Jones, R. M., Somerville, L. H., Li, J., Ruberry, E. J., Powers, A., Mehta, N., .. Casey, B. J. (2014). Adolescent-specific patterns of behavior and neural activity during social reinforcement learning. Cognitive, Affective \& Behavioral Neuroscience, 14(2), 683697. https://doi.org/10.3758/s13415-014-0257-z

Kanwisher, N., McDermott, J., \& Chun, M. M. (1997). The Fusiform Face Area: A Module in Human Extrastriate Cortex Specialized for Face Perception. Journal of Neuroscience, 17(11), 4302-4311.

Kasper, L., Bollmann, S., Diaconescu, A. O., Hutton, C., Heinzle, J., Iglesias, S., ... Stephan, K. E. (2017). The PhysIO Toolbox for Modeling Physiological Noise in fMRI Data. Journal of Neuroscience Methods. https://doi.org/10.1016/j.jneumeth.2016.10.019

Keramati, M., Smittenaar, P., Dolan, R. J., \& Dayan, P. (2016). Adaptive integration of habits into depth-limited planning defines a habitual-goal-directed spectrum. Proceedings of the National Academy of Sciences of the United States of America. https://doi.org/10.1073/pnas.1609094113 
Kessler, R. C., Berglund, P., Demler, O., Jin, R., Merikangas, K. R., \& Walters, E. E. (2005). Lifetime prevalence and age-of-onset distributions of DSM-IV disorders in the National Comorbidity Survey Replication. Archives of General Psychiatry, 62(6), 593-602. https://doi.org/10.1001/archpsyc.62.6.593

Kidd, C., \& Hayden, B. Y. (2015). The Psychology and Neuroscience of Curiosity. Neuron, 88(3), 449-460. https://doi.org/10.1016/j.neuron.2015.09.010

Krystal, J. H., Anticevic, A., Yang, G. J., Dragoi, G., Driesen, N. R., Wang, X.-J., \& Murray, J. D. (2017). Impaired Tuning of Neural Ensembles and the Pathophysiology of Schizophrenia: A Translational and Computational Neuroscience Perspective. $\begin{array}{llr}\text { Biological Psychiatry, } & \text { 81(10), } & \text { 874-885. }\end{array}$ https://doi.org/10.1016/j.biopsych.2017.01.004

Kumar, P., Waiter, G., Ahearn, T., Milders, M., Reid, I., \& Steele, J. D. (2008). Abnormal temporal difference reward-learning signals in major depression. Brain: A Journal of Neurology, 131(Pt 8), 2084-2093. https://doi.org/10.1093/brain/awn136

Kurniawan, I. T., Guitart-Masip, M., Dayan, P., \& Dolan, R. J. (2013). Effort and valuation in the brain: the effects of anticipation and execution. The Journal of Neuroscience: The Official Journal of the Society for Neuroscience, 33(14), 6160-6169. https://doi.org/10.1523/JNEUROSCI.4777-12.2013

Kurth-Nelson, Z., Economides, M., Dolan, R. J., \& Dayan, P. (2016). Fast Sequences of Nonspatial State Representations in Humans. Neuron, $O(0)$. https://doi.org/10.1016/j.neuron.2016.05.028

Ladd, G. W., \& Troop-Gordon, W. (2003). The role of chronic peer difficulties in the development of children's psychological adjustment problems. Child Development, 74(5), 1344-1367. 
Leary, M. R., Tambor, E. S., Terdal, S. K., \& Downs, D. L. (1995). Self-Esteem as an Interpersonal Monitor: The Sociometer Hypothesis. Journal of Personality and Social Psychology, 68, 518-530.

Marin, R. S. (1991). Apathy: a neuropsychiatric syndrome. The Journal of Neuropsychiatry and Clinical Neurosciences, 3(3), 243-254. https://doi.org/10.1176/jnp.3.3.243

Marr, D. (1982). Vision: A Computational Investigation Into the Human Representation and Processing of Visual Information. Cambridge, Mass: MIT Press.

Montague, P. R., Dayan, P., \& Sejnowski, T. J. (1996). A framework for mesencephalic dopamine systems based on predictive hebbian learning. The Journal of Neuroscience, 16(5), 1936-1947.

Montague, P. R., Dolan, R. J., Friston, K. J., \& Dayan, P. (2012). Computational psychiatry. Trends in Cognitive Sciences, 16(1), 72-80. https://doi.org/10.1016/j.tics.2011.11.018

Murray, G. K., Corlett, P. R., Clark, L., Pessiglione, M., Blackwell, A. D., Honey, G., ... Fletcher, P. C. (2007). Substantia nigra/ventral tegmental reward prediction error disruption in psychosis. Molecular Psychiatry, 13(3), 267-276. https://doi.org/10.1038/sj.mp.4002058

Naneix, F., Marchand, A. R., Di Scala, G., Pape, J.-R., \& Coutureau, E. (2012). Parallel maturation of goal-directed behavior and dopaminergic systems during adolescence. The Journal of Neuroscience: The Official Journal of the Society for Neuroscience, 32(46), 16223-16232. https://doi.org/10.1523/JNEUROSCI.3080-12.2012

O’Dea, J. A., \& Abraham, S. (2000). Improving the body image, eating attitudes, and behaviors of young male and female adolescents: a new educational approach that focuses on selfesteem. The International Journal of Eating Disorders, 28(1), 43-57. 
O’Doherty, J. P., Dayan, P., Friston, K., Critchley, H., \& Dolan, R. J. (2003). Temporal difference models and reward-related learning in the human brain. Neuron, 38(2), 329337.

O’Doherty, J. P., Dayan, P., Schultz, J., Deichmann, R., Friston, K., \& Dolan, R. J. (2004). Dissociable roles of ventral and dorsal striatum in instrumental conditioning. Science (New York, N.Y.), 304(5669), 452-454. https://doi.org/10.1126/science.1094285

Orth, U., \& Robins, R. W. (2013). Understanding the Link Between Low Self-Esteem and Depression. Current Directions in Psychological Science, 22(6), 455-460. https://doi.org/10.1177/0963721413492763

Palminteri, S., Kilford, E. J., Coricelli, G., \& Blakemore, S.-J. (2016). The Computational Development of Reinforcement Learning during Adolescence. PLOS Computational Biology, 12(6), e1004953. https://doi.org/10.1371/journal.pcbi.1004953

Paus, T. (2001). Primate anterior cingulate cortex: Where motor control, drive and cognition interface. Nature Reviews. Neuroscience, 2(6), 417-424. https://doi.org/10.1038/35077500

Paus, T. (2010). Growth of white matter in the adolescent brain: myelin or axon? Brain and Cognition, 72(1), 26-35. https://doi.org/10.1016/j.bandc.2009.06.002

Pessiglione, M., Seymour, B., Flandin, G., Dolan, R. J., \& Frith, C. D. (2006). Dopaminedependent prediction errors underpin reward-seeking behaviour in humans. Nature, 442(7106), 1042-1045. https://doi.org/10.1038/nature05051

Pessiglione, M., Vinckier, F., Bouret, S., Daunizeau, J., \& Le Bouc, R. (2017). Why not try harder? Computational approach to motivation deficits in neuro-psychiatric diseases. Brain: A Journal of Neurology. https://doi.org/10.1093/brain/awx278 
Potter, T. C. S., Bryce, N. V., \& Hartley, C. A. (2017). Cognitive components underpinning the development of model-based learning. Developmental Cognitive Neuroscience, 25, 272-280. https://doi.org/10.1016/j.dcn.2016.10.005

Reiter, A. M. F., Deserno, L., Kallert, T., Heinze, H.-J., Heinz, A., \& Schlagenhauf, F. (2016). Behavioral and Neural Signatures of Reduced Updating of Alternative Options in Alcohol-Dependent Patients during Flexible Decision-Making. The Journal of Neuroscience: The Official Journal of the Society for Neuroscience, 36(43), 1093510948. https://doi.org/10.1523/JNEUROSCI.4322-15.2016

Reynolds, L. M., Pokinko, M., Torres-Berrío, A., Cuesta, S., Lambert, L. C., Pellitero, E. D. C., ... Flores, C. (2018). DCC Receptors Drive Prefrontal Cortex Maturation by Determining Dopamine Axon Targeting in Adolescence. Biological Psychiatry, 83(2), 181-192. https://doi.org/10.1016/j.biopsych.2017.06.009

Roberts, J. E., Shapiro, A. M., \& Gamble, S. A. (1999). Level and perceived stability of selfesteem prospectively predict depressive symptoms during psychoeducational group treatment. The British Journal of Clinical Psychology, 38 ( Pt 4), 425-429.

Rodman, A. M., Powers, K. E., \& Somerville, L. H. (2017). Development of self-protective biases in response to social evaluative feedback. Proceedings of the National Academy of Sciences of the United States of America, 114(50), 13158-13163. https://doi.org/10.1073/pnas.1712398114

Rutledge, R. B., Dean, M., Caplin, A., \& Glimcher, P. W. (2010). Testing the reward prediction error hypothesis with an axiomatic model. The Journal of Neuroscience: The Official Journal of the Society for Neuroscience, 30(40), 13525-13536. https://doi.org/10.1523/JNEUROSCI.1747-10.2010

Rutledge, R. B., Moutoussis, M., Smittenaar, P., Zeidman, P., Taylor, T., Hrynkiewicz, L., ... Dolan, R. J. (2017). Association of Neural and Emotional Impacts of Reward Prediction 
Errors With Major Depression. JAMA Psychiatry, 74(8), 790-797. https://doi.org/10.1001/jamapsychiatry.2017.1713

Schultz, W., Dayan, P., \& Montague, P. R. (1997). A neural substrate of prediction and reward. Science, 275(5306), 1593-1599. https://doi.org/10.1126/science.275.5306.1593

Seymour, B., O’Doherty, J. P., Dayan, P., Koltzenburg, M., Jones, A. K., Dolan, R. J., ... Frackowiak, R. S. (2004). Temporal difference models describe higher-order learning in humans. Nature, 429(6992), 664-667. https://doi.org/10.1038/nature02581

Seymour, B., O’Doherty, J. P., Koltzenburg, M., Wiech, K., Frackowiak, R., Friston, K., \& Dolan, R. (2005). Opponent appetitive-aversive neural processes underlie predictive learning of pain relief. Nature Neuroscience, 8(9), 1234-1240. https://doi.org/10.1038/nn1527

Silver, D., Huang, A., Maddison, C. J., Guez, A., Sifre, L., van den Driessche, G., ... Hassabis, D. (2016). Mastering the game of Go with deep neural networks and tree search. Nature, 529(7587), 484-489. https://doi.org/10.1038/nature16961

Somerville, L. H., Sasse, S. F., Garrad, M. C., Drysdale, A. T., Abi Akar, N., Insel, C., \& Wilson, R. C. (2017). Charting the expansion of strategic exploratory behavior during adolescence. Journal of Experimental Psychology. General, 146(2), 155-164. https://doi.org/10.1037/xge0000250

Sowislo, J. F., \& Orth, U. (2013). Does low self-esteem predict depression and anxiety? A meta-analysis of longitudinal studies. Psychological Bulletin, 139(1), 213-240. https://doi.org/10.1037/a0028931

Sundström, E., Kölare, S., Souverbie, F., Samuelsson, E. B., Pschera, H., Lunell, N. O., \& Seiger, A. (1993). Neurochemical differentiation of human bulbospinal monoaminergic neurons during the first trimester. Brain Research. Developmental Brain Research, $75(1), 1-12$. 
Sutton, R. S., \& Barto, A. G. (1998). Reinforcement learning: An introduction. MIT Press.

van den Bos, W., Bruckner, R., Nassar, M. R., Mata, R., \& Eppinger, B. (2017). Computational neuroscience across the lifespan: Promises and pitfalls. Developmental Cognitive Neuroscience. https://doi.org/10.1016/j.dcn.2017.09.008

van den Bos, W., Cohen, M. X., Kahnt, T., \& Crone, E. A. (2012). Striatum-medial prefrontal cortex connectivity predicts developmental changes in reinforcement learning. Cerebral Cortex (New York, N.Y.: 1991), 22(6), 1247-1255. https://doi.org/10.1093/cercor/bhr198

van Harmelen, A.-L., Hauber, K., Gunther Moor, B., Spinhoven, P., Boon, A. E., Crone, E. A., \& Elzinga, B. M. (2014). Childhood emotional maltreatment severity is associated with dorsal medial prefrontal cortex responsivity to social exclusion in young adults. PloS One, 9(1), e85107. https://doi.org/10.1371/journal.pone.0085107

Virchow, R. (1854). Ueber das ausgebreitete Vorkommen einer dem Nervenmark analogen Substanz in den thierischen Geweben. Archiv für pathologische Anatomie und Physiologie und für klinische Medicin, 6(4), 562-572. https://doi.org/10.1007/BF02116709

Walton, M. E., Bannerman, D. M., Alterescu, K., \& Rushworth, M. F. S. (2003). Functional specialization within medial frontal cortex of the anterior cingulate for evaluating effort-related decisions. The Journal of Neuroscience: The Official Journal of the Society for Neuroscience, 23(16), 6475-6479.

Will, G.-J., Rutledge, R. B., Moutoussis, M., \& Dolan, R. J. (2017). Neural and computational processes underlying dynamic changes in self-esteem. ELife, 6. https://doi.org/10.7554/eLife.28098

Will, G.-J., van Lier, P. A. C., Crone, E. A., \& Güroğlu, B. (2016). Chronic Childhood Peer Rejection is Associated with Heightened Neural Responses to Social Exclusion During 
Adolescence. Journal of Abnormal Child Psychology, 44(1), 43-55. https://doi.org/10.1007/s10802-015-9983-0

Wilson, R. C., Geana, A., White, J. M., Ludvig, E. A., \& Cohen, J. D. (2014). Humans use directed and random exploration to solve the explore-exploit dilemma. Journal of Experimental Psychology. General, 143(6), 2074-2081. https://doi.org/10.1037/a0038199

Wunderlich, K., Dayan, P., \& Dolan, R. J. (2012). Mapping value based planning and extensively trained choice in the human brain. Nature Neuroscience, 15(5), 786-791. https://doi.org/10.1038/nn.3068

Wunderlich, K., Smittenaar, P., \& Dolan, R. J. (2012). Dopamine enhances model-based over model-free choice behavior. Neuron, 75(3), 418-424. https://doi.org/10.1016/j.neuron.2012.03.042

Yamins, D. L. K., Hong, H., Cadieu, C. F., Solomon, E. A., Seibert, D., \& DiCarlo, J. J. (2014). Performance-optimized hierarchical models predict neural responses in higher visual cortex. Proceedings of the National Academy of Sciences of the United States of America, 111(23), 8619-8624. https://doi.org/10.1073/pnas.1403112111

Ziegler, G., Hauser, T. U., Moutoussis, M., Bullmore, E. T., Goodyer, I. M., Fonagy, P., ... Dolan, R. J. (under review). Compulsivity and impulsivity are linked to distinct aberrant developmental trajectories of fronto-striatal myelination.

Zipser, D., \& Andersen, R. A. (1988). A back-propagation programmed network that simulates response properties of a subset of posterior parietal neurons. Nature, 331(6158), 679684. https://doi.org/10.1038/331679a0 